\title{
Biopanning of mouse bone marrow mesenchymal stem cell affinity for cyclic peptides
}

\author{
GUOZONG WANG ${ }^{1,2}$, ZHENTAO MAN $^{1}$, NIANPING ZHANG ${ }^{3}$,

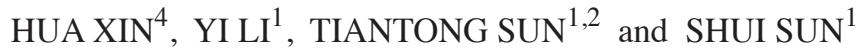 \\ ${ }^{1}$ Department of Joint Surgery, Shandong Provincial Hospital Affiliated to Shandong University, Jinan, \\ Shandong 250021; ${ }^{2}$ College of Clinical Medicine, Shandong University, Jinan, Shandong 250012; \\ ${ }^{3}$ The Teaching and Research Section of Surgery, The First Clinical College of Shandong University \\ of Traditional Chinese Medicine, Jinan, Shandong 250355; ${ }^{4}$ Department of Neurology, \\ People's Hospital of Rizhao, Rizhao, Shandong 222000, P.R. China
}

Received March 7, 2018; Accepted October 16, 2018

DOI: $10.3892 / \mathrm{mmr} .2018 .9626$

\begin{abstract}
Osteonecrosis of the femoral head (ONFH) is a refractory disease present worldwide. In the development of therapies for this disease, mesenchymal stem cells (MSC) are a promising candidate cell source in tissue engineering (TE) and regenerative medicine. MSCs harvested from bone marrow (BM) are the gold standard. A significant barrier for BMMSC-based therapies is the inability and decreased number of BMMSCs in the tissues of interest. The ability to recruit BMMSCs efficiently to defective or injured sites in tissues or organs, for example the necrotic area of the femoral head in vivo, has been a major concern. In the present study, a peptide sequence (CDNVAQSVC), termed D7, was identified through phage display technology using C57BL/6 mouse BMMSCs. Subsequent analysis suggested that the identified loop-constrained heptapeptide exhibited a high specific affinity for mouse BMMSCs. Due to this specific affinity for BMMSCs, the present study provides a selective method to improve MSC-based TE strategies for the treatment of ONFH.
\end{abstract}

\section{Introduction}

Osteonecrosis of the femoral head (ONFH) is a progressive disease that may cause collapse of the femoral head, pain and gait disorders. During the continuous disease progression, collapse of the femoral head eventually leads to secondary osteoarthritis of the hip joint. The incidence of collapse of the femoral head

Correspondence to: Professor Shui Sun, Department of Joint Surgery, Shandong Provincial Hospital Affiliated to Shandong University, 324 Jingwuweiqi Road, Huaiyin, Jinan, Shandong 250021, P.R. China

E-mail: sunshui1965@foxmail.com

Key words: phage display, affinity cyclic peptide, bone marrow mesenchymal stem cell, recruitment, osteonecrosis of the femoral head and osteoarthritis in untreated patients is $70-80 \%$ (1). The disease usually affects young adults, and $5-12 \%$ of patients require total hip arthroplasty (2). It has been demonstrated that engraftment therapy of autologous bone marrow may be used as a method of treatment (3). Mesenchymal stem cell (MSC) treatment has also received much interest.

MSCs have the ability to differentiate into a number of cell types, including osteoblasts, adipocytes and chondrocytes (4). Studies involving MSCs are progressively developing towards clinical application phases. MSCs are the most commonly utilized cell type in tissue engineering (TE) fields (5). For example, in order to treat avascular necrosis of the femoral head, skeletal stem cells and impaction bone grafting were combined to produce a mechanically stable living bone composite (6). MSCs have been isolated from various tissues including bone marrow (BM), adipose tissue, dental pulp, hair follicle, cornea, umbilical cord and placenta (7). The current gold standard is MSCs that have been harvested from BM (5). BMMSCs may be isolated and cultured from animals and humans. A total of 4 randomized trials demonstrated decreased time to collapse, improvements in pain score and decreased lesion sizes with core decompression and BMMSCs treatment, compared with core decompression alone to cure ONFH (8-11).

The most significant barrier for MSC-based therapies is the decreased number of MSCs in the tissues of interest. Given the necrotic nature of $\mathrm{ONFH}$, the lesions associated with osteonecrosis have poor innate regenerative capacity with few or no viable MSCs (5).

A number of attempts have been made to solve this problem. Among these applied methods, the strategy of affinity peptides of BMMSCs obtained through phage display technology is popular (12). Phage display is a selection technique in which a library of peptide or protein variants is expressed on the outside of a phage virion, while the genetic material encoding each variant resides on the inside. In order to recruit the seed cells and elevate the treatment efficiency, a large number of biological materials have been modified using these affinity peptides (12-18).

In the present study, an affinity peptide of mouse bone marrow-derived MSCs was acquired using the phage display 

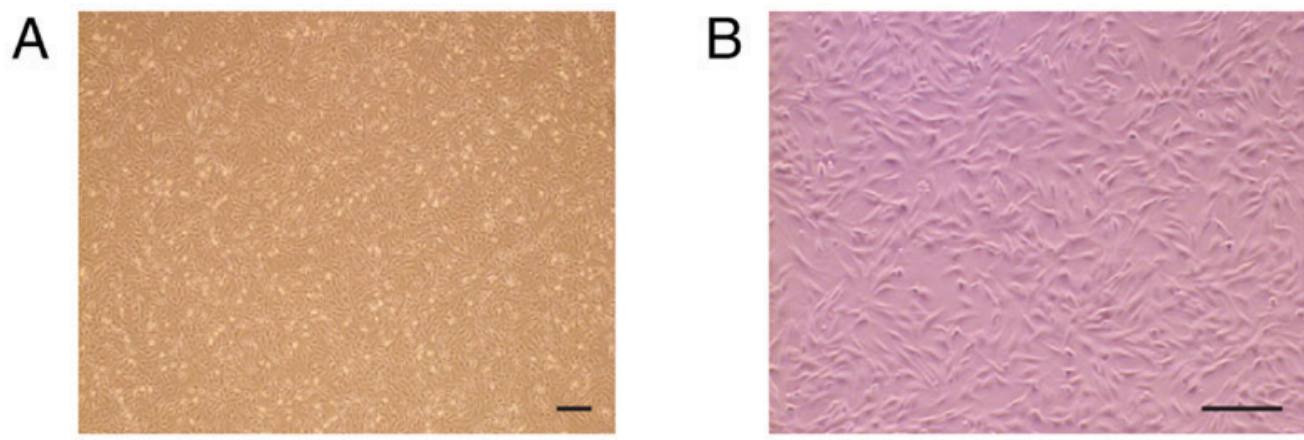

Figure 1. C57BL/6 mouse BMMSCs cultures. The C57BL/6 mouse BMMSCs at passage 3 exhibited a spindle-shaped or fibroblastic appearance. (A) Images are at magnification, $x 40$. (B) Images are at magnification, $x 100$. Scale bars $=250 \mu \mathrm{m}$. BMMSCs, bone marrow mesenchymal stem cells.

technique. The aim was to conjugate this peptide on the surface of materials. As peptide sequences have a certain level of conservation in different species, we hypothesize that the materials modified by the affinity peptide may be applied to the therapy of the ONFH in TE and increase the adhesive efficiency of BMMSCs (12).

\section{Materials and methods}

Cellculture.C57BL/6mouseBMMSCs (cat.no.MUBMX-01001) were obtained from Cyagen Biosciences, Inc., (Santa Clara, CA, USA). The cells were cultured in cell culture flasks in a humidified atmosphere with $5 \% \mathrm{CO}_{2}$ at $37^{\circ} \mathrm{C}$ (Fig. 1). Cells were cultured in low-glucose Dulbecco's modified Eagle's medium (DMEM; Biological Industries, Ltd., Kibbutz Beit-Haemek, Israel; cat. no. 01-051-1A) with L-glutamine containing 10\% fetal bovine serum (Gibco; Thermo Fisher Scientific, Inc., Waltham, MA, USA; cat. no., 10099141) and antibiotics [100 U/ml penicillin and $0.1 \mathrm{mg} / \mathrm{ml}$ streptomycin $(\mathrm{P} / \mathrm{S})$; Gibco; Thermo Fisher Scientific, Inc.; cat. no. 15140122]. The medium was changed every 2-3 days. Cells were dissociated and passaged at 80-90\% confluence. Mouse BMMSCs at passage 4-5 were used for subsequent experiments.

Phage display biopanning. Phage display biopanning, an affinity selection technique that selects peptides through binding to a given target, was performed according to a previously described protocol, with certain modifications (14). Peptide sequences with an affinity for C57BL/6 mouse bone marrow-derived MSCs were identified by screening the Ph.D.C7C ${ }^{\text {тм }}$ Phage Display Library (New England Biolabs, Inc., Ipswich, MA, USA; cat. no., E8120S) against clonally-derived mouse BMMSCs at passage 4-5. The phage display library consisted of $10^{9}$ different phages. Cells plated in 60x15 mm petri dishes were rinsed twice with PBS (Biological Industries, Ltd.; cat. no., 02-024-1A) and pre-blocked with DMEM containing $0.5 \%$ BSA (albumin bovine fraction-V; NeoFroxx, Einhausen, Germany; cat. no., GRM105-5G) without supplements at $37^{\circ} \mathrm{C}$ and $5 \% \mathrm{CO}_{2}$ for $1 \mathrm{~h}$. The Ph.D.C7C ${ }^{\text {тм }}$ Phage Display Library ( $\left.1 \times 10^{11} \mathrm{PFU}\right)$ was then introduced to the cells. Non-binding phages were then discarded, and the cells were washed 5 times in cold PBS. The phages binding to the cells were eluted with $1 \mathrm{ml}$ Glycine/ $\mathrm{HCl}$ (pH 2.2) with $1 \mathrm{mg} / \mathrm{ml} \mathrm{BSA}$ for $20 \mathrm{~min}$ at room temperature while being gently shaken. The eluted phages were collected and neutralized with $0.15 \mathrm{ml}$
$1 \mathrm{M}$ Tris- $\mathrm{HCl}$ (pH 9.1). A total of 3 rounds of panning were performed for each sample.

Sequencing. Following 3 rounds of panning, blue plaques were randomly selected for sequencing. Sequencing of phage DNA was performed by GENEWIZ, Inc., (Suzhou, China). Firstly, the phage DNA was enriched using the rolling circle amplification technique. This technique uses Phi29 DNA polymerase and random hexamer primers to amplify circular DNA molecules. Subsequently, the amplified phage DNA products were used for Sanger sequencing. The bioinformatics analysis of the generated data was carried out using Chromas 2.6.5 software (Technelysium Pty Ltd., South Brisbane, Australia).

Synthesis of peptides. A specific peptide was identified in the C57BL/6 mouse BMMSC-affinity clones and designated as D7. The peptide was a loop-constrained heptapeptide with a pair of cysteine residues conjugated to each terminus of 7 amino acids. The two cysteine residues formed an intramolecular disulfide linkage. A peptide (Aa sequence CVAVQNDSC) with the same amino acids as D7 in a scrambled order was used as the negative control and designated as V7. A peptide comprising 3 amino acids (arginine, glycine and aspartic acid) was used as the positive control and termed RGD. All the peptides were synthesized through solid-phase peptide synthesis using fluorenylmethyloxyccarbonyl chemistry (Scilight-Peptide, Inc., Beijing, China). An extra 6-aminocaproic acid molecule was attached at the amino $(\mathrm{N})$ terminal of all peptides to facilitate fluorescein-5-isothiocyanate (FITC) labeling. The FITC-labeled peptides were stored in $-20^{\circ} \mathrm{C}$. A $1 \mathrm{mg} / \mathrm{ml}$ concentration was obtained by dissolving the peptides in PBS prior to use.

Peptide-affinity assay via flow cytometry. The C57BL/6 mouse BMMSCs were washed twice with PBS, and dissociated with $0.25 \%$ trypsin-EDTA (Gibco; Thermo Fisher Scientific, Inc.). The cell suspension was then centrifuged at $250 \mathrm{x} g$ for $5 \mathrm{~min}$ at room temperature to collect cell sedimentation. The cells were incubated with $10 \mu \mathrm{M}$ FITC-labeled peptides (Beijing Scilight-Peptide Ltd., Co.) for $1 \mathrm{~h}$ at $37^{\circ} \mathrm{C}$ to allow cell binding and internalization. The mouse BMMSC affinity properties of the peptides were analyzed quantitatively using flow cytometry (BD LSR Fortessa; Becton, Dickinson and Company, Franklin Lakes, NJ, USA) at a wavelength of $488 \mathrm{~nm}$ and FlowJo 7.6.1 software (Tree Star, Inc., Ashland, OR, USA). 
Table I. Recovery efficiency of phage display biopanning increases round by round.

\begin{tabular}{lcccc}
\hline Round no. & Input titer, pfu & Output titer, pfu & Recovery efficiency & Fold increase \\
\hline 1 & $1.0 \times 10^{11}$ & $5.175 \times 10^{4}$ & $5.175 \times 10^{-7}$ & 1 \\
2 & $1.0 \times 10^{11}$ & $1.84 \times 10^{6}$ & $1.84 \times 10^{-5}$ & 35.6 \\
3 & $1.0 \times 10^{11}$ & $2.3 \times 10^{6}$ & $2.3 \times 10^{-5}$ & 44.4 \\
\hline
\end{tabular}

The recovery efficiency was calculated as the output titer divided by the input titer.

Peptide-affinity assay via fluorescence cytochemistry. The C57BL/6 mouse BMMSCs were cultured in 24-well dishes until 70-90\% confluence was achieved. Then, the cells were incubated with $10 \mu \mathrm{M}$ FITC-labeled peptides for $1 \mathrm{~h}$ at $37^{\circ} \mathrm{C}$. The mouse BMMSCs were also incubated with $160 \mathrm{nM}$ rhodamine phalloidin (Beijing Solarbio Science and Technology, Inc., Beijing, China) for $30 \mathrm{~min}$ at room temperature to visualize the cytoskeleton. The nuclei were then counterstained with $10 \mu \mathrm{g} / \mathrm{ml}$ DAPI (Beijing Solarbio Science and Technology, Inc.) for $10 \mathrm{~min}$ at room temperature. The cells on the round coverslips were examined under a fluorescence microscope at magnification, $\mathrm{x} 200$.

Binding efficiency by competition assay. The affinity peptide was synthesized by a company (Scilight-Peptide, Ltd., Co.). A total of $7 \times 10^{5}$ C57BL/6 mouse BMMSCs were cultured on $60 \times 15 \mathrm{~mm}$ petri dishes until 70-90\% confluence was reached. Then, $1 \times 10^{9}$ phages with the D7 sequence were added to the cells. The synthetic peptide was introduced to cells at a concentration of $100 \mu \mathrm{M}$ prior to the addition of the phages during the process of biopanning, as aforementioned. The level of binding of phages to mouse BMMSCs was calculated comparing the output titer of phages selected with the competition of the affinity peptide with the output titer of phages selected without the addition of the affinity peptide.

Statistical analysis. In the present study, data is expressed as mean \pm standard deviation. A one-way analysis of variance was performed to evaluate the differences between multiple groups followed by Dunnett's post-hoc test using IBM SPSS Statistics 24.0 software (IBM Corp., Armonk, NY, USA). P<0.05 was considered to indicate a statistically significant difference.

\section{Results}

Recovery efficiency following biopanning increases round by round. Following each round of biopanning, the recovery efficiencies of the specific phage clones were calculated as the output titer divided by the input titer of the phages. The input titer of the phages was $1 \times 10^{11}$ PFU for three rounds. As indicated in Table I, the best recovery efficiency was attained in the third round, which was 44.4-fold higher compared with that in the first round. The phage clones remaining after the third round of biopanning were selected for peptide sequencing.

Peptide sequencing. The C57BL/6 mouse BMMSC affinity phage clones were isolated following three rounds of phage display biopanning. Subsequent to these three rounds,
Table II. Peptide sequences.

\begin{tabular}{llc}
\hline Round no. & Peptide sequence & Expression, \% $(\mathrm{n}=10)$ \\
\hline 1 & Not sequenced & - \\
2 & Not sequenced & - \\
3 & DNVAQSV & 60 \\
& KTSPWAK & 10 \\
& Empty phage & 30 \\
\hline
\end{tabular}

10 blue plaques were randomly selected for sequencing. With the exception of empty phages, there was one type of phage clone that appeared 6 times. Using DNA sequencing, the sequence, DNVAQSV, was identified via Sanger sequencing applying Rolling Circle Amplification technique and Chromas analysis software (Table II and Fig. 2A). Corresponding cyclic peptides (Fig. 2B) indicated a high affinity for the mouse BMMSCs through the subsequent experiments.

Identified loop-constrained heptapeptide has a high specific affinity for mouse BMMSCs. The identified peptide (CDNVAQSVC) was designated as a mouse BMMSCs affinity peptide (D7). The molecular weight of the linear precursor of D7 peptide was determined to be 939.54 Da by mass spectrometry (Fig. 2C). The molecular weight of D7 peptide decreased to $937.90 \mathrm{Da}$ (Fig. 2D). This indicated that the thiol groups of the two cysteine residues formed an intramolecular disulfide linkage, and that the cyclic peptide D7 was successfully synthesized. A peptide (CVAVQNDSC) with the same amino acids as D7 in a scrambled order was used as the negative control and termed V7. RGD, a peptide composed of 3 amino acids (arginine, glycine and aspartic acid), was used as the positive control. The C57BL/6 mouse BMMSCs were incubated for $1 \mathrm{~h}$ with FITC-labeled D7, V7 or RGD, and measured via flow cytometry. The average fluorescence intensity was $4,059.0 \pm 213.5$ for the mouse BMMSCs incubated with FITC-D7, 3,058.5 \pm 604.6 for the mouse BMMSCs incubated with FITC-RGD, and 146.5 \pm 60.1 for the mouse BMMSCs incubated with FITC-V7. The average fluorescence intensity for the mouse BMMSCs incubated with FITC-D7 and FITC-RGD was increased than that of FITC-V7, and the difference was statistically significant $(n=3$; $\mathrm{P}<0.01$; Figs. 3 and 4). The average fluorescence intensity of cells incubated with FITC-D7 was 31.2-fold higher compared with that of the FITC-V7 cells. The cells were also observed under a fluorescence microscope. Marked fluorescent signals 
A
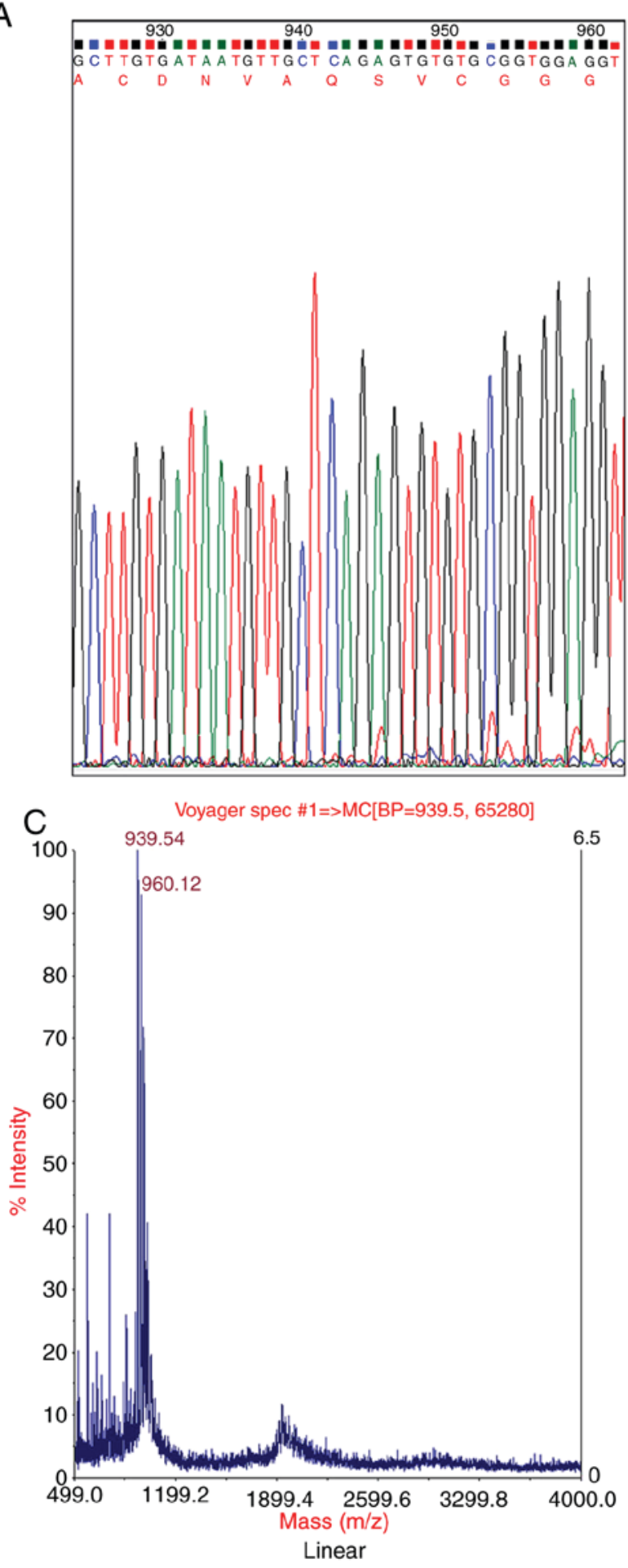

B

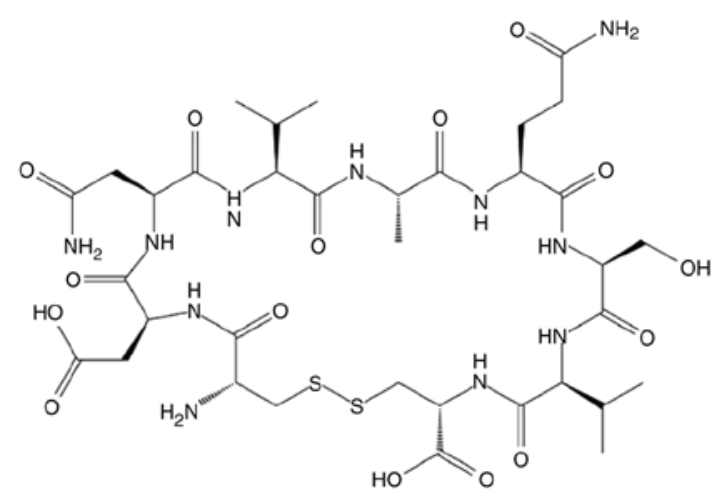

Cys-Asp-Asn-Val-Ala-GIn-Ser-Val-Cys

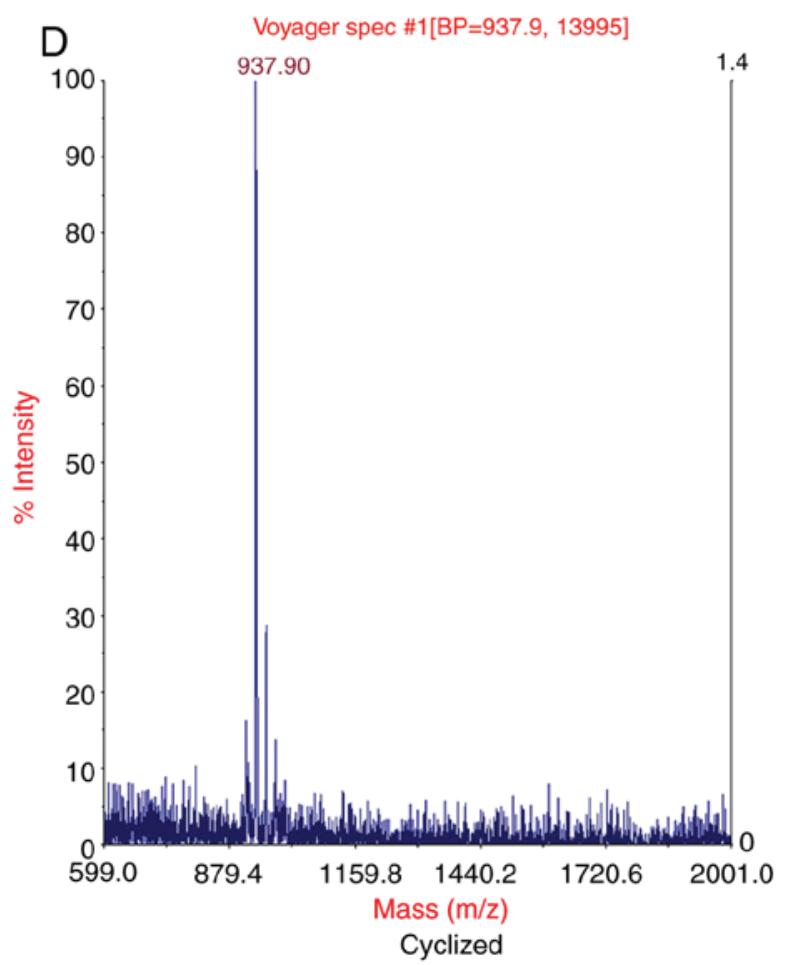

Figure 2. A peptide sequence is successfully identified using phage display technology. (A) Following 3 rounds of phage display biopanning a peptide sequence (CDNVAQSVC), which has a high specific affinity for C57BL/6 mouse BMMSCs, was identified by DNA sequencing. The peptide sequence was obtained using Chromas analysis software. (B) The peptide is a loop-constrained heptapeptide with a pair of cysteine residues conjugated to each terminus of the 7 amino acids (DNVAQSV). The 2 cysteine residues formed an intramolecular disulfide linkage. (C) The molecular weight of the linear precursor of D7 peptide was determined to be 939.54 Da by mass spectrometry. (D) The molecular weight of D7 peptide decreased to 937.90 Da due to the formation of an intramolecular disulfide linkage.

were observed in the cells incubated with FITC-D7 and FITC-RGD, whereas weak or no fluorescent signals were observed in the cells incubated with FITC-V7 (Fig. 5). These results suggested that D7 exhibited a high affinity for mouse BMMSCs.
No significant difference is identified in the affinity peptide competition assay. A synthetic peptide containing the CDNVAQSVC sequence competing with phages for the binding of C57BL/6 mouse BMMSCs was examined. In the experiment, the phages expressed the loop-constrained 


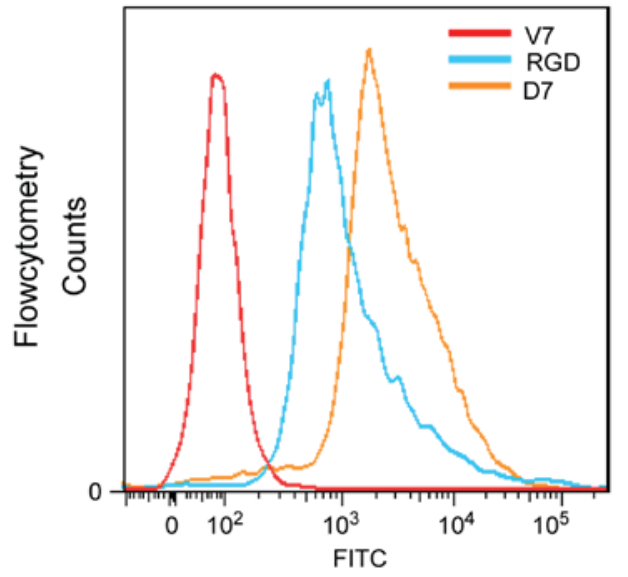

Figure 3. Identified cyclic peptide D7 demonstrates a high affinity for C57BL/6 mouse BMMSCs by flow cytometry. Affinity of the D7 peptide for C57BL/6 mouse BMMSCs was investigated via flow cytometry. The cells were incubated with FITC-labeled D7 (FITC-D7), RGD (FITC-RGD), and V7 (FITC-V7) and the fluorescence was analyzed by flow cytometry. The peaks of the experimental group (D7) and the positive control group (RGD) were shifted markedly to the right compared with the negative control group (V7), which indicated that the cyclic peptide D7 exhibited a good affinity for the target cells. D7, CDNVAQSVC; V7, CVAVQNDSC; BMMSCs, bone marrow mesenchymal stem cells; FITC, fluorescein isothiocyanate; RGD, arginine, glycine and aspartic acid.

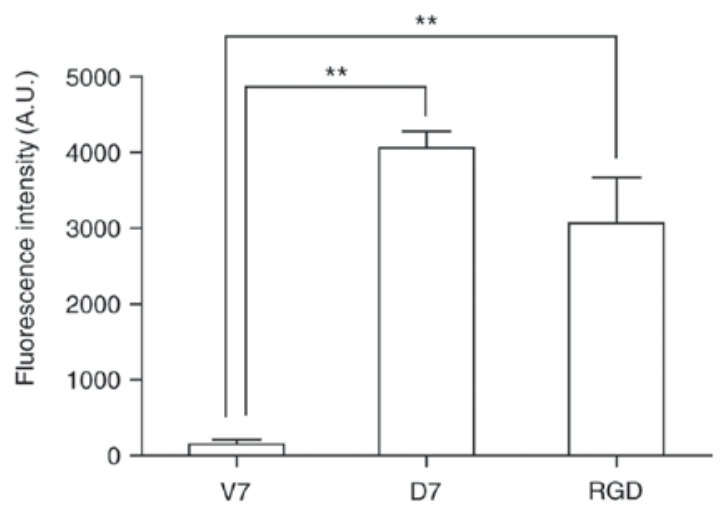

Figure 4. Affinity of the identified cyclic peptide D7 towards C57BL/6 mouse BMMSCs is quantitatively analyzed by flow cytometry. The affinity of the peptide D7 towards C57BL/6 mouse BMMSCs was analyzed quantitatively via flow cytometry following incubation of FITC-V7, FITC-D7 and FITC-RGD. The average fluorescence intensity for the cells incubated with FITC-D7 and FITC-RGD was increased compared with that of FITC-V7, and the difference was statistically significant. Data is expressed as mean \pm standard deviation $(\mathrm{n}=3) .{ }^{* *} \mathrm{P}<0.01$ vs. V7. D7, CDNVAQSVC; V7, CVAVQNDSC; BMMSCs, bone marrow mesenchymal stem cells; FITC, fluorescein isothiocyanate; RGD, arginine, glycine and aspartic acid; A.U., arbitrary units.

D7 peptide on the outside. Using $100 \mu \mathrm{M}$ D7 peptide, the following results were obtained. The output titer of phages selected with the competition of the affinity peptide was $4.14 \times 10^{4}$, while the output titer of phages selected without adding the affinity peptide was $4.025 \times 10^{4}$. It was identified that there was no significant difference in the binding of phages to target cells. A total of 3 independent experiments were performed and the same trend was observed in each repeat. This was consistent with the phenomenon of the appearance of non-loaded phages following 3 rounds of panning.

\section{Discussion}

In the present study, following 3 rounds of biopanning, empty phages appeared. The enrichment of insertless clones is not unusual. The Ph.D. libraries were constructed by cloning a randomized DNA insert into a phage vector, so all of the libraries contain a small percentage $(0.1-0.5 \%)$ of clones with no insert. Insertless clones begin to predominate panning experiments if an excessive number of rounds of amplification have been performed. In the case of the $\mathrm{C} 7 \mathrm{C}$ library, the presence of the 2 cysteines in the peptide sufficiently attenuated phage propagation such that the empty vector had a growth advantage. Concomitantly, the disulfide linkage in the $\mathrm{C} 7 \mathrm{C}$ increased the affinity of peptide sequences that bind the target in a conformation allowed by the disulfide constraint. Therefore, the clones containing the insert that were present following 3 rounds of biopanning were considered to be optimal for subsequent analysis. In the present study, the insertless clones were disregarded in favor of the clones containing the insert.

In phage display biopanning, as unconjugated phages are gradually eliminated, the recovery efficiencies should increase. In the present study, following 3 rounds of biopanning, the recovery efficiencies increased round by round. The recovery efficiency increased by 44.4-fold following 3 rounds of screening. These data confirmed that at this point, the phages binding to C57BL/6 mouse BMMSCs were effectively enriched.

The lack of effective therapy for ONFH is a difficulty to be overcome in clinical practice, and the clinical results of traditional methods have not been satisfactory. At present, TE strategies are being investigated. One of the most common strategies currently used in TE is the combination of a biodegradable matrix (scaffold), exogenous or endogenous living cells and/or biologically active molecules to form a construct that promotes tissue repair and regeneration. BMMSCs are considered to be a good cell source for bone regeneration, due to their high proliferation rates and good osteogenic potential, as demonstrated by a number of previous studies $(6,19,20)$. However, there are few or no viable MSCs in necrotic femoral head sites (5). Additionally, several previous studies have indicated that only a small percentage of stem cells remain at the injury site after 2-3 weeks post-injection $(21,22)$. Therefore, promoting the precise and efficient homing and entrapment of stem cells into injured tissues to form 'stem cell nests' should be a primary aim in TE therapy. Abundant BMMSCs are required in order to build 'stem cell niches', to constantly facilitate repair during the entire regeneration process. However, the problems of homing and entrapment arise when BMMSCs are adopted as a cell source for bone repair and regeneration. Therefore, the low efficiency of homing and entrapment of MSCs becomes a key problem to be overcome in endogenous stem cell-based TE, and recruitment of endogenous BMMSCs to the injured sites becomes important.

A number of methods have been examined to solve this problem. Recruiting BMMSCs using affinity peptides is popular. Biomaterials modified by affinity peptides for target cells including BMMSCs have been studied increasingly. Phage display provides a novel method for searching for affinity peptides highly specific to BMMSCs. For example, a peptide sequence (DPIYALSWSGMA) with high affinity for 


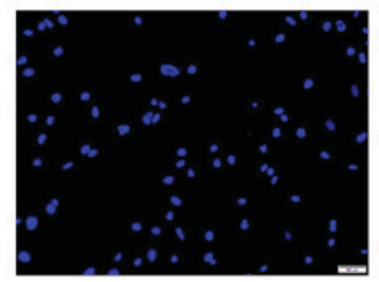

Nuclei

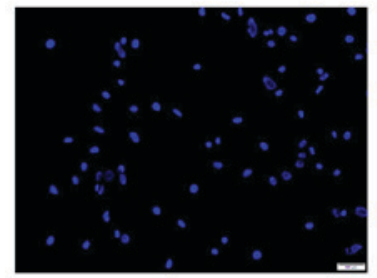

Nuclei

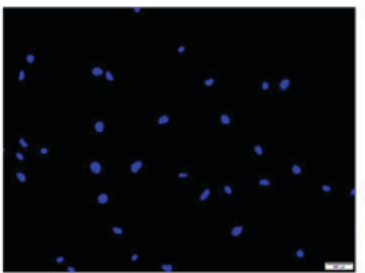

Nuclei

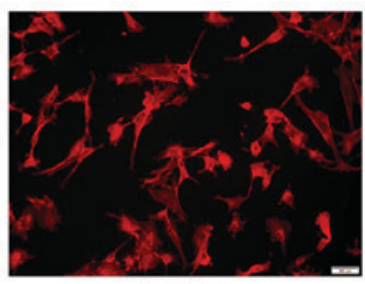

Phalloidin

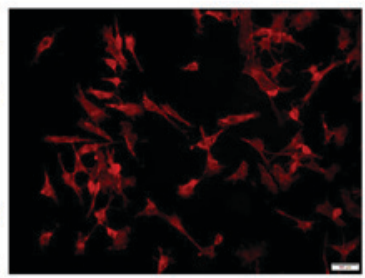

Phalloidin

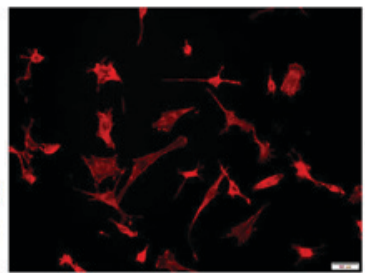

Phalloidin

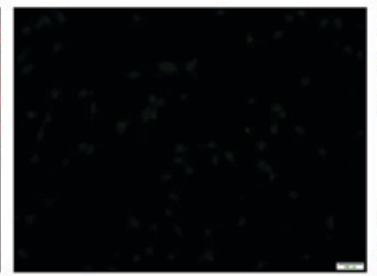

V7-FITC

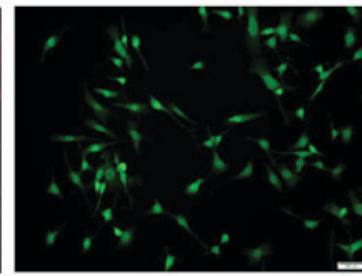

D7-FITC

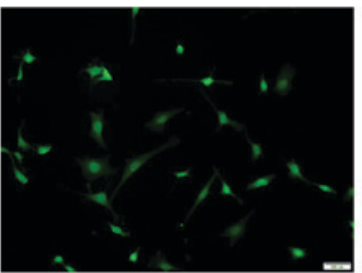

RGD-FITC

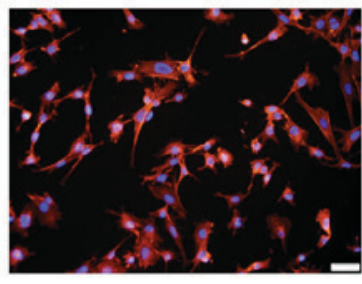

Merge

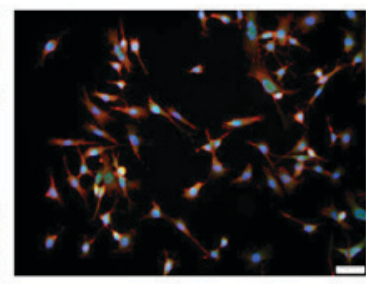

Merge

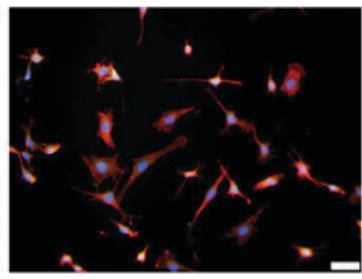

Merge

Figure 5. Identified cyclic peptide D7 exhibits high affinity for C57BL/6 mouse BMMSCs by fluorescence cytochemistry. Affinity of the cyclic peptide D7 towards C57BL/6 mouse BMMSCs was investigated via fluorescence staining. The cells incubated with FITC-D7, FITC-RGD and FITC-V7 were observed under a fluorescence microscope. Marked FITC signals were observed in cells incubated with FITC-D7 and FITC-RGD, whereas weak or no signals were observed in cells incubated with FITC-V7. The nuclei were stained with DAPI, and the cytoskeleton was stained with rhodamine phalloidin. Scale bars $=50 \mu \mathrm{m}$. D7, CDNVAQSVC; V7, CVAVQNDSC; BMMSCs, bone marrow mesenchymal stem cells; FITC, fluorescein isothiocyanate; RGD, arginine, glycine and aspartic acid.

human BMMSCs, was identified using phage display technology (14). A novel peptide with the amino acid sequence of EPLQLKM (E7) with high specific affinity for BMMSCs was also identified via phage display technology. The E7 peptide was covalently conjugated onto polycaprolactone electrospun meshes to fabricate functional scaffolds to cure cartilage defect of rat knee joints (12). E7 peptides were also conjugated to demineralized bone matrix to create functional biomaterials combined with chitosan solution to cure the osteochondral defect (23). RGD is best known for cell adhesion (24). The peptide derives from fibronectin in the extracellular matrix. As the RGD peptide was identified to promote cell adhesion in 1984 (25), a number of materials modified by RGD have been applied for academic studies or clinical therapies (26-28). However, this peptide lacks specificity, as fibronectin receptors exist in all cells. In the present study, the cyclic peptide D7 identified may also be used to modify appropriate materials to fabricate functional scaffolds. In addition, the disulfide linkage in the cyclic peptide will increase the affinity of peptide sequences that bind the target in a conformation allowed by the disulfide constraint.

However, the exact mechanism of this affinity peptide was not investigated in the present study. Therefore, future studies will aim to identify the receptor of the affinity peptide on the surface of mouse BMMSCs and explore the potential mechanism. Core decompression is recommended as the first surgical treatment option in symptomatic small to medium-sized pre-collapsed lesions of ONFH (29). A bone tunnel from the greater trochanter to the femoral head is built, decreasing intraosseous pressure and pain. In light of the age of the patient population, this type of treatment represents a reasonable initial surgical intervention. Core decompression has been combined with a number of bone-graft substitutes that are filled into the bone tunnel to treat ONFH. A previous study described the effect of core decompression combined with calcium phosphate composite scaffolds containing bone morphogenetic protein-vascular endothelial growth factor-loaded poly-lactic-co-glycolic acid microspheres in the treatment of ONFH (30). Therefore, future studies will aim to select appropriate materials and modify them using the affinity cyclic peptide identified in the present study to fabricate functional biomaterials. Then, core decompression will be combined with these functional biomaterials to recruit endogenous BMMSCs and increase the efficiency of TE therapy in ONFH.

In conclusion, through phage display panning technology, the D7 peptide sequence, a loop-constrained heptapeptide with a pair of cysteine residues conjugated to each terminus of 7 amino acids, was identified. The peptide exhibited a specific affinity for C57BL/6 mouse BMMSCs. Subsequent in vitro experiments further confirmed the high affinity of the D7 peptide for mouse BMMSCs. These data suggest that the D7 peptide can be used as a potent motif for recruitment of BMMSCs in MSC-based TE therapy. 


\section{Acknowledgements}

Not applicable.

\section{Funding}

The present study was supported by the National Natural Science Foundation of China (grant no., 81271966).

\section{Availability of data and materials}

The data sets generated and analyzed during the current study are available from the corresponding author on reasonable request.

\section{Authors' contributions}

GW, ZM and SS designed the experiments. GW performed the experiments. GW, NZ, HX, YL and TS analyzed the data. GW wrote the manuscript. GW revised the manuscript. All authors reviewed the manuscript.

\section{Ethics approval and consent to participate}

Not applicable.

\section{Patient consent for publication}

Not applicable.

\section{Competing interests}

The authors declare that they have no competing interests.

\section{References}

1. Hernigou P, Poignard A and Nogier A: Fate of very small asymptomatic stage-I osteonecrotic lesions of the hip. J Bone Joint Surg Am 86-A: 2589-2593, 2004.

2. Mont MA, Carbone JJ and Fairbank AC: Core decompression versus nonoperative management for osteonecrosis of the hip. Clin Orthop Relat Res: 169-178, 1996.

3. Hernigou P, Flouzat-Lachaniette CH, Delambre J, Poignard A, Allain J, Chevallier N and Rouard H: Osteonecrosis repair with bone marrow cell therapies: State of the clinical art. Bone 70 102-109, 2015.

4. Pittenger MF, Mackay AM, Beck SC, Jaiswal RK, Douglas R, Mosca JD, Moorman MA, Simonetti DW, Craig S and Marshak DR: Multilineage potential of adult human mesenchymal stem cells. Science 284: 143-147, 1999.

5. Tatara AM: Tissue engineering in orthopaedics. J Bone Joint Surg Am 98: 1132-1139, 2016.

6. Aarvold A, Smith JO, Tayton ER, Jones AM, Dawson JI, Lanham S, Briscoe A, Dunlop DG and Oreffo RO: A tissue engineering strategy for the treatment of avascular necrosis of the femoral head. Surgeon 11: 319-325, 2013.

7. Hass R, Kasper C, Böhm S and Jacobs R: Different populations and sources of human mesenchymal stem cells (MSC): A comparison of adult and neonatal tissue-derived MSC. Cell Commun Signal 9: 12, 2011.

8. Zhao D, Cui D, Wang B, Tian F, Guo L, Yang L, Liu B and Yu X: Treatment of early stage osteonecrosis of the femoral head with autologous implantation of bone marrow-derived and cultured mesenchymal stem cells. Bone 50: 325-330, 2012.

9. Sen RK, Tripathy SK, Aggarwal S, Marwaha N, Sharma RR and Khandelwal N: Early results of core decompression and autologous bone marrow mononuclear cells instillation in femoral head osteonecrosis: A randomized control study. J Arthroplasty 27: 679-686, 2012.
10. Gangji V, De Maertelaer V and Hauzeur JP: Autologous bone marrow cell implantation in the treatment of non-traumatic osteonecrosis of the femoral head: Five year follow-up of a prospective controlled study. Bone 49: 1005-1009, 2011.

11. Lim YW, Kim YS, Lee JW and Kwon SY: Stem cell implantation for osteonecrosis of the femoral head. Exp Mol Med 45: e61, 2013.

12. Shao Z, Zhang X, Pi Y, Wang X, Jia Z, Zhu J, Dai L, Chen W, Yin L, Chen $\mathrm{H}$, et al: Polycaprolactone electrospun mesh conjugated with an MSC affinity peptide for MSC homing in vivo. Biomaterials 33: 3375-3387, 2012.

13. Meng Q, Man Z, Dai L, Huang H, Zhang X, Hu X, Shao Z, Zhu J, Zhang J, Fu X, et al: A composite scaffold of MSC affinity peptide-modified demineralized bone matrix particles and chitosan hydrogel for cartilage regeneration. Sci Rep 5: 17802, 2015.

14. Ramaraju H, Miller SJ and Kohn DH: Dual-functioning peptides discovered by phage display increase the magnitude and specificity of BMSC attachment to mineralized biomaterials. Biomaterials 134: 1-12, 2017.

15. Li Q, Xing D, Ma L and Gao C: Synthesis of E7 peptide-modified biodegradable polyester with the improving affinity to mesenchymal stem cells. Mater Sci Eng C Mater Biol Appl 73: 562-568, 2017.

16. Man Z, Yin L, Shao Z, Zhang X, Hu X, Zhu J, Dai L, Huang H, Yuan L,Zhou C, et al: The effects of co-delivery of BMSC-affinity peptide and rhTGF- $\beta 1$ from coaxial electrospun scaffolds on chondrogenic differentiation. Biomaterials 35: 5250-5260, 2014.

17. Shao Z, Zhang X, Pi Y, Yin L, Li L, Chen H, Zhou C and Ao Y: Surface modification on polycaprolactone electrospun mesh and human decalcified bone scaffold with synovium-derived mesenchymal stem cells-affinity peptide for tissue engineering. J Biomed Mater Res A 103: 318-329, 2015.

18. Pi Y, Zhang X, Shi J, Zhu J, Chen W, Zhang C, Gao W, Zhou C and Ao Y: Targeted delivery of non-viral vectors to cartilage in vivo using a chondrocyte-homing peptide identified by phage display. Biomaterials 32: 6324-6332, 2011.

19. Udehiya RK, Amarpal, Aithal HP, Kinjavdekar P, Pawde AM, Singh R and Taru Sharma G: Comparison of autogenic and allogenic bone marrow derived mesenchymal stem cells for repair of segmental bone defects in rabbits. Res Vet Sci 94: 743-752, 2013.

20. Chen M, Le DQ, Kjems J, Bünger C and Lysdahl H: Improvement of distribution and osteogenic differentiation of human mesenchymal stem cells by hyaluronic acid and $\beta$-tricalcium phosphate-coated polymeric scaffold in vitro. Biores Open Access 4: 363-373, 2015.

21. Discher DE, Mooney DJ and Zandstra PW: Growth factors, matrices, and forces combine and control stem cells. Science 324 1673-1677, 2009.

22. Karp JM and Leng Teo GS: Mesenchymal stem cell homing: The devil is in the details. Cell Stem Cell 4: 206-216, 2009.

23. Huang HJ, Zhang X, Hu X, Shao Z, Zhu J, Dai L, Man Z, Yuan L, Chen H, Zhou C and Ao Y: A functional biphasic biomaterial homing mesenchymal stem cells for in vivo cartilage regeneration. Biomaterials 35: 9608-9619, 2014.

24. Ruoslahti E and Pierschbacher MD: Arg-Gly-Asp: A versatile cell recognition signal. Cell 44: 517-518, 1986.

25. Pierschbacher MD and Ruoslahti E: Cell attachment activity of fibronectin can be duplicated by small synthetic fragments of the molecule. Nature 309: 30-33, 1984.

26. Hersel U, Dahmen C and Kessler H: RGD modified polymers: Biomaterials for stimulated cell adhesion and beyond. Biomaterials 24: 4385-4415, 2003.

27. Zhang $\mathrm{H}$ and Hollister S: Comparison of bone marrow stromal cell behaviors on poly(caprolactone) with or without surface modification: Studies on cell adhesion, survival and proliferation. J Biomater Sci Polym Ed 20: 1975-1993, 2009.

28. Zhang H, Lin CY and Hollister SJ: The interaction between bone marrow stromal cells and RGD-modified three-dimensional porous polycaprolactone scaffolds. Biomaterials 30: 4063-4069, 2009.

29. Mont MA, Cherian JJ, Sierra RJ, Jones LC and Lieberman JR: Nontraumatic osteonecrosis of the femoral head: Where do we stand today? A ten-year update. J Bone Joint Surg Am 97: 1604-1627, 2015.

30. Zhang HX, Zhang XP, Xiao GY, Hou Y, Cheng L, Si M, Wang SS, $\mathrm{Li} \mathrm{YH}$ and Nie L: In vitro and in vivo evaluation of calcium phosphate composite scaffolds containing BMP-VEGF loaded PLGA microspheres for the treatment of avascular necrosis of the femoral head. Mater Sci Eng C Mater Biol Appl 60: 298-307, 2016.

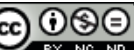

This work is licensed under a Creative Commons Attribution-NonCommercial-NoDerivatives 4.0 International (CC BY-NC-ND 4.0) License. 